[4] Remennik, V. V. (2000). Development of managerial decisions. Moscow: UNITY, 140.

[5] Feinberg, A. (1976). Problems of analysis and decision-making procedures. Moscow: Mir, 126-145.

[6] Abdrahimov, D., Ioffin, A. Universal information-analytical system (IAS) decision support "for evaluating and selecting”. Razrabotchyky YAS “Ocenka y vyybor”. Available at: http://www.deol.ru/users/ DecisionSupporter/projects/iasctc.html

[7] The Ministry of Industrial Policy is drafting engineering development for (2012-2017). BAU.ua. Available at: http://news.bau.ua.

[8] Statistichni danni promislovosti Ukraine. Derzhstat Ukrai'ny. Available at: http://www.ukrstat.gov.ua

[9] Hrustalev, E. M. Aggregation of data in OLAP-cubes. Analytical systems.

[10] Hrustalev, E. M. Information technology. iTeam. Available: http://www.iteam.ru/publications/ it/section_92/article_1759.

[11] Mark, D., McGowan, K. (1993). Methodology of structural analysis and design. Moscow: Meta.

[12] Schekochikhin, O. V., Shvedenko, V. V. (2006). Simulation management solutions in an object-functional business management system. Izvestiya of the Tula State University. Tula: TSU, 117-123.

[13] Saaty, T. L. (1986). Axiomatic Foundation of the Analytic Hierarchy Process. Management Science, Vol. 32, № 7, 841-855. doi:http://doi.org/10.1287/mnsc.32.7.841

\title{
ADVANTAGES OF OPTICAL ORTHOGONAL FREQUENCY DIVISION MULTIPLEXING IN COMMUNICATIONS SYSTEMS
}

\author{
Ali Abdourahamane \\ Department of Telecommunication Systems and networks \\ Kharkiv national university of radio electronics \\ 14 Lenin ave, Kharkiv, Ukraine, 61166 \\ alzabrmaowy@gmail.com
}

\begin{abstract}
The role of the optical transmitter is to generate the optical signal, impose the information bearing signal, and launch the modulated signal into the optical fiber. The semiconductor light sources are commonly used in state-of-theart optical communication systems. Optical communication systems has become one of the important systems after the advent of telephone, internet, radio networks in the second half of the 20 -th century. The development of optical communication was caused primarily by the rapidly rising demand for Internet connectivity. Orthogonal frequency-division multiplexing (OFDM) belongs to a wide class of multicarrier modulation. Orthogonal frequency-division multiplexing has succeeded in a wide range of applications in the wireless communication domain from video/audio digital broadcasting to wireless local area networks (LANs). Although their very low loss compared to that of the wireless counterpart, optical systems still need renovation for spans commonly less than $150 \mathrm{~km}$. In this paper advantages of optical orthogonal frequency division multiplexing in communications systems will explained.
\end{abstract}

Keywords: OFDM, WDM, optical signal, communication systems, radio networks.

(C) Ali Abdourahamane

\section{Introduction}

Communication systems transmit information from a transmitter to a receiver through the construction of a time-varying physical quantity or a signal. Optical communication systems use light rays with a wavelength of $380 \mathrm{~nm}$ to $3000 \mathrm{~nm}$ region of the electromagnetic spectrum. The spectral efficiency and reliability of wireless optical channels can also be improved by using multiple transmitter and receiver elements after the invention of the optical amplifier in the 1990-s. The advent of the optical amplifier heralded a new era of optical communications in which a massive number of wavelength-division multiplexing (WDM) signals can be conveyed over thousands of kilometers [1]. Fiber optic communications are light wave systems that employ optical fibers for data transmission. The natural trend was to study the light wave communication systems, in which 
the data rate can be increased dramatically. This was boosted after the invention and the realization of a laser that gives a coherent source for the transmitter [2]. Amplified systems came into use in the early 1990-s and instantly boosted the capacity via supporting WDM systems. By 2003, the $40 \mathrm{~Tb} / \mathrm{s}$ per channel wavelength-division multiplexing system was commercially deployed. Recent research demonstrates transmission of $21.7 \mathrm{~Tb} / \mathrm{s}$, thereby crossing the $10 \mathrm{~Tb} / \mathrm{s}$ per fiber barrier [3, 4]. The concept of orthogonal frequency-division multiplexing OFDM was first introduced by Chang in a seminal paper in 1966. The term "OFDM" in fact first appeared in a separate patent of his in 1970 [5]. In 1995 Orthogonal frequency-division multiplexing was adopted as the European DAB standard, ensuring its significance as an important modulation technology and heralding a new era of OFDM success in a broad range of applications. The application of OFDM to optical communications occurred surprisingly late and relatively scantly compared with the RF counterpart, although the same acronym of OFDM has long been used to stand for "optical frequency division multiplexing" in the optical communications community $[6,7]$. The first paper on optical OFDM in the open literature was reported by Pan and Green in 1996 [8], and there was also some intermittent research on optical OFDM over the ensuing years $[8,9]$. However, the fundamental advantage of orthogonal frequency-division multiplexing, namely, its robustness against optical channel dispersion was not recognized in optical communications until 2001, when Dixon et al. 9 proposed the use of OFDM to combat modal dispersion in multimode fiber. Given the fact that the multimode fiber channel resembles that of the wireless channel in terms of multipath fading, it is not surprising that the early body of work on optical OFDM concentrated on multimode fiber application $[10,11]$.The increased interest in optical OFDM is in large part attributed to independent proposals of optical OFDM for long-haul applications from three groups, including direct-detection optical OFDM and coherent optical-OFDM [12].

\section{The objective of the paper}

Internet is the main cause of the recent explosion of activity in optical fiber telecommunications. The high growth rates observed on the Internet, and the popular perception that growth rates were even higher, led to an upsurge in research, development, and investment in telecommunications.

Orthogonal frequency-division multiplexing also has intrinsic disadvantages, such as high peak-to-average power ratio (PAPR) and sensitivity to frequency and phase noise. Therefore, a proper understanding of orthogonal frequency-division multiplexing basics is essential for the study of its applications in the emerging field of optical orthogonal frequency-division multiplexing.

Also fundamental advantages of orthogonal frequency-division multiplexing are its robustness against channel dispersion and its ease of phase and channel estimation in a time-varying environment with the advancement of powerful silicon technology.

\section{Orthogonal Frequency-Division Multiplexing (OFDM) Basics}

Mathematical Formulation of an OFDM Signal:

Orthogonal frequency-division multiplexing (OFDM) is a special class of multicarrier modulation, a generic implementation of which is depicted in Fig. 1. The structure of a complex multiplier (IQ modulator/demodulator), which is commonly used in multicarrier modulation systems, is also shown in the Fig. 1. The multicarrier modulation transmitted signal s(t) is represented as

$$
\begin{gathered}
\mathrm{s}(\mathrm{t})=\sum_{\mathrm{i}=-\infty}^{+\infty} \sum_{\mathrm{k}=1}^{\mathrm{N}_{\mathrm{sc}}} \operatorname{ckisk}\left(\mathrm{t}-\mathrm{iT}_{\mathrm{S}}\right), \\
\mathrm{s}(\mathrm{t})=\prod(\mathrm{t}) \mathrm{e}^{\mathrm{j} 2 \pi \mathrm{kt} \mathrm{t}} \\
\prod(\mathrm{t})=\left\{\begin{array}{l}
1,\left(0<\mathrm{i} \leq \mathrm{T}_{\mathrm{s}}\right) \\
0,\left(\mathrm{t} \leq 0, \mathrm{t}>\mathrm{T}_{\mathrm{s}}\right.
\end{array}\right.
\end{gathered}
$$


where $c_{k i}$ is the $\mathrm{i}$-th information symbol at the $\mathrm{k}$-th subcarrier, $\mathrm{s}_{\mathrm{k}}$ is the waveform for the $\mathrm{k}_{\mathrm{th}}$ subcarrier, $\mathrm{N}_{\mathrm{sc}}$ is the number of subcarriers, $\mathrm{f}_{\mathrm{k}}$ is the frequency of the subcarrier, Ts is the symbol period, and $\Pi(\mathrm{t})$ is the pulse shaping function. The optimum detector for each subcarrier could use a filter that matches the subcarrier waveform or a correlator matched to the subcarrier as shown in Fig. 1.

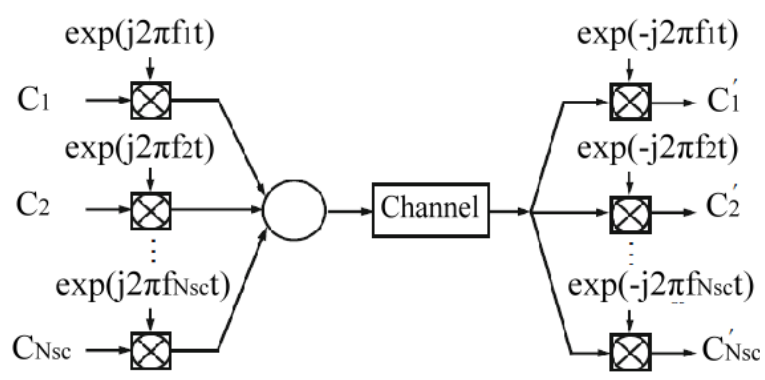

Fig. 1. Conceptual diagram for a generic multicarrier modulation system

Therefore, the detected information symbol $c_{i k}$ at the output of the correlator is given by

$$
c_{k i}^{\prime}=\frac{1}{T_{S}} \int_{0}^{T_{S}} r\left(t-i T_{s}\right) S_{k}^{*} d t=\frac{1}{T_{S}} \int_{0}^{T_{S}} r\left(t-i T_{s}\right) e^{-j 2 \pi f k t} d t
$$

where $r(t)$ is the received time domain signal. The classical multicarrier modulation uses nonoverlapped band limited signals and can be implemented with a bank of large numbers of oscillators and filters at both transmit and receive ends [13]. The major disadvantage of multicarrier modulation is that it requires excessive bandwidth. This is because to design the filters and oscillators cost-effectively, the channel spacing has to be a multiple of the symbol rate, greatly reducing the spectral efficiency. A novel approach, OFDM, was investigated by employing overlapped yet orthogonal signal sets [14]. This orthogonality originates from a straightforward correlation between any two subcarriers, given by

$$
\delta_{k l}=\frac{1}{T_{s}} \int_{0}^{T_{s}} s_{k} s^{*}{ }_{l} d t=\frac{1}{T_{s}} \int_{0}^{T_{s}} \exp \left(j 2 \pi\left(f_{k}-f_{l}\right) t\right) d t=\exp \left(j \pi\left(f_{k}-f_{l}\right) T_{s}\right) \frac{\sin \left(\pi\left(f_{k}-f_{l}\right) T_{s}\right)}{\pi\left(f k-f_{l}\right) T_{s}} .
$$

It can be seen that if the condition

$$
\mathrm{f}_{\mathrm{k}}-\mathrm{f}_{\mathrm{l}}=\mathrm{m} \frac{1}{\mathrm{~T}_{\mathrm{s}}}
$$

is satisfied, then the two subcarriers are orthogonal to each other. This signifies that these orthogonal subcarrier sets, with their frequencies spaced at multiples of inverse of the symbol periods, can be recovered with the matched filters in Eq. (4) without intercarrier interference (ICI), despite strong signal spectral overlapping.

\section{Spectral Efficiency for Optical OFDM}

In direct-detection optical-OFDM systems, the optical spectrum is usually not a linear replica of the RF spectrum; therefore, the optical spectral efficiency is dependent on the detailed implementation. We turn our attention to the optical spectral efficiency for coherent optical-OFDM systems. In coherent optical-OFDM systems, $\mathrm{N}_{\mathrm{sc}}$ subcarriers are transmitted in every OFDM symbol period of Ts. Thus, the total symbol rate $\mathrm{R}$ for coherent optical-OFDM systems is given by

$$
\mathrm{R}=\mathrm{N}_{\mathrm{sc}} / \mathrm{T}_{\mathrm{S}} \text {. }
$$

Fig. 2, $\boldsymbol{a}$ shows the spectrum of wavelength-division multiplexed (WDM) channels, each with coherent optical - OFDM modulation, and Fig. 2, $\boldsymbol{b}$ shows the zoomed-in optical spectrum 
for each wavelength channel. We use the bandwidth of the first null to denote the boundary of each wavelength channel. The OFDM bandwidth, $\mathrm{B}_{\mathrm{OFDM}}$, is thus given by

$$
\mathrm{B}_{\text {OFDM }}=\frac{2}{\mathrm{~T}_{\mathrm{s}}}+\frac{\mathrm{N}_{\mathrm{sc}}-1}{\mathrm{t}_{\mathrm{s}}}
$$

where $t_{s}$ is the observation period. Assuming that a large number of subcarriers are used, the bandwidth efficiency of OFDM $\eta$ is found to be

$$
\eta=2 \frac{\mathrm{R}}{\mathrm{B}_{\text {OFDM }}}=2 \alpha, \alpha=\frac{\mathrm{t}_{\mathrm{s}}}{\mathrm{T}_{\mathrm{s}}}
$$

The factor of 2 accounts for two polarizations in the fiber. Using a typical value of 8/9, we obtain the optical spectral efficiency factor of $1.8 \mathrm{Bd} / \mathrm{Hz}$. The optical spectral efficiency gives $3.6 \mathrm{bit} / \mathrm{s} / \mathrm{Hz}$ if quaternary phase-shift keying modulation is used for each subcarrier. The spectral efficiency can be further improved by using higher order QAM modulation $[15,16]$. To practically implement coherent optical-OFDM systems, the optical spectral efficiency will be reduced due to the need for a sufficient guard band between WDM channels, taking account of laser frequency drift of approximately $2 \mathrm{GHz}$. This guard band can be avoided by using orthogonality across the WDM channels, which is discussed in the next section.

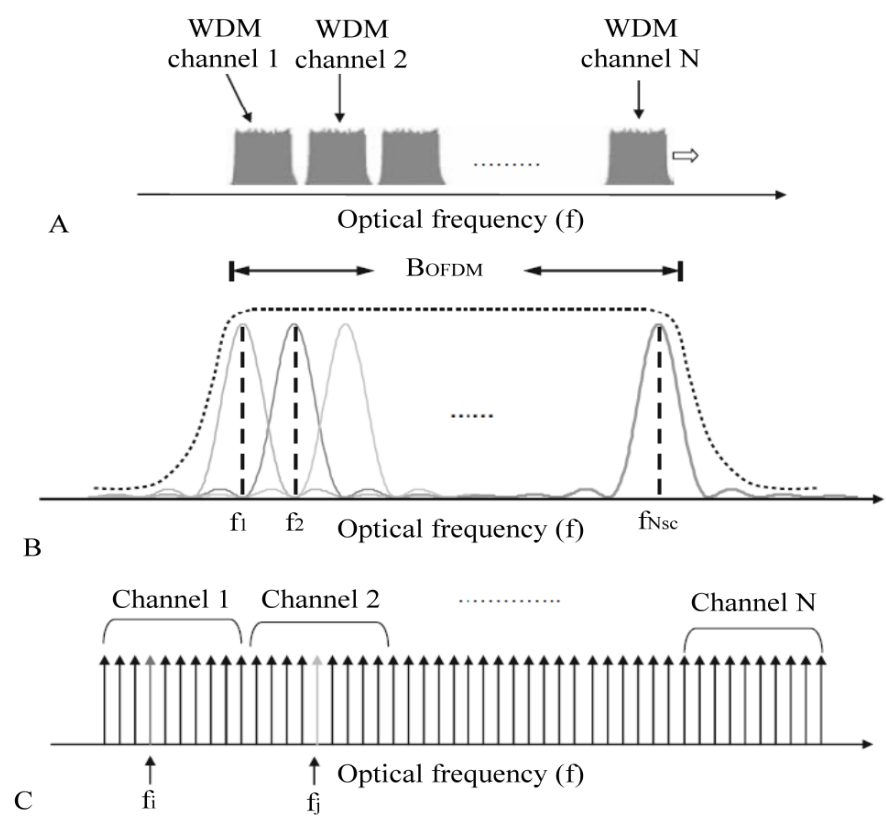

Fig. 2. Optical spectra for: A - N wavelength-division multiplexed (WDM) CO-OFDM channels, B - zoomed-in OFDM signal for one wavelength, and C - cross-channel OFDM (XC-OFDM) without guard band

\section{The Difference between RF OFDM and Optical OFDM Systems}

It is a fallacy that because RF OFDM has been extensively studied during approximately the past 20 years, optical OFDM will be an effortless one-to-one translation from the wireless domain to the optical domain. As we shall see in what follows, a clear understanding of the uniqueness of the optical channel and the optical systems makes possible the most efficient design of the coherent optical-OFDM systems. Using the SMF optical communication systems and the wireless systems as examples, we lay out the following differences that have significant ramifications for the OFDM design. A typical wireless channel can be modeled as summarization of the multiple paths that each undergoes a Rayleigh process, given by $[17,18]$. 


$$
\mathrm{h}(\mathrm{t}, \tau)=\sum_{\mathrm{l}=1}^{\mathrm{L}} \mathrm{a}_{1} \cdot \mathrm{g}_{1}(\mathrm{t}) \cdot \delta\left(\tau-\tau_{1}\right)
$$

where al is a complex constant, $g_{1}(t)$ is the Rayleigh fading process, and $t_{1}$ is the delay for the 1-th path. A optical SMF can be more conveniently modeled in the frequency domain for the two polarization components in the fiber expressed as [19].

$$
\begin{gathered}
H(t, f)=e^{j \phi_{D}(f)} \cdot T_{k}, \\
\phi_{D}(f)=\pi \cdot c \cdot D_{t} \cdot \frac{f^{2}}{f_{L D 1}^{2}} \\
T_{k}=\prod_{l=1}^{N} \exp \left(\left(-\frac{1}{2} j \cdot \vec{\beta}_{1}(t) \cdot f-\frac{1}{2} \vec{\alpha}_{1}(t) \cdot \vec{\sigma}\right),\right.
\end{gathered}
$$

where $\varphi_{D}(f)$ is the phase dispersion due to the fiber chromatic dispersion effect, $T_{k}$ is the Jones matrix for the fiber link representing the polarization-dependent effect including PMD and polarization dependent loss (PDL), $\mathrm{N}$ is the number of PMD/PDL cascading elements represented by their birefringence vector $\vec{\beta}_{1}$ and PDL vector $\vec{\alpha}_{1}$, [20] and $\vec{\sigma}$ is the Pauli matrix vector [20]. The significance of the channel model is that the majority of the channel dispersion $\mathrm{e}^{\mathrm{j} \mathrm{\phi}_{\mathrm{D}}(\mathrm{f})}$ can be first estimated and factored out for the channel estimation. Dynamic dispersion comes from the Jones matrix $T_{k}$, but it can be reduced effectively to a summation of only a few taps of the FIR model if its mean PMD value is known. Therefore, dependent on the PMD value and the data rate, the channel estimation can be greatly simplified for the optical OFDM systems [21]. In the wireless systems, because of the scarcity of the spectrum, the RF channel is packed as tightly as possible. Therefore, there is a stringent out-of-band emission requirement that is enforced upon the OFDM transmitter. Fig. 3 shows the transmission mask for a Wi-Fi signal that details the maximum relative intensity to which the transmission emission should be restricted [22]. For instance, for the Wi-Fi signal with channel spacing of $20 \mathrm{MHz}$, the maximum out-of-band emission intensity at 11,20 , and $30 \mathrm{MHz}$ is, respectively, $-20,=28$, and $-30 \mathrm{~dB}$ relative to the in-band density.

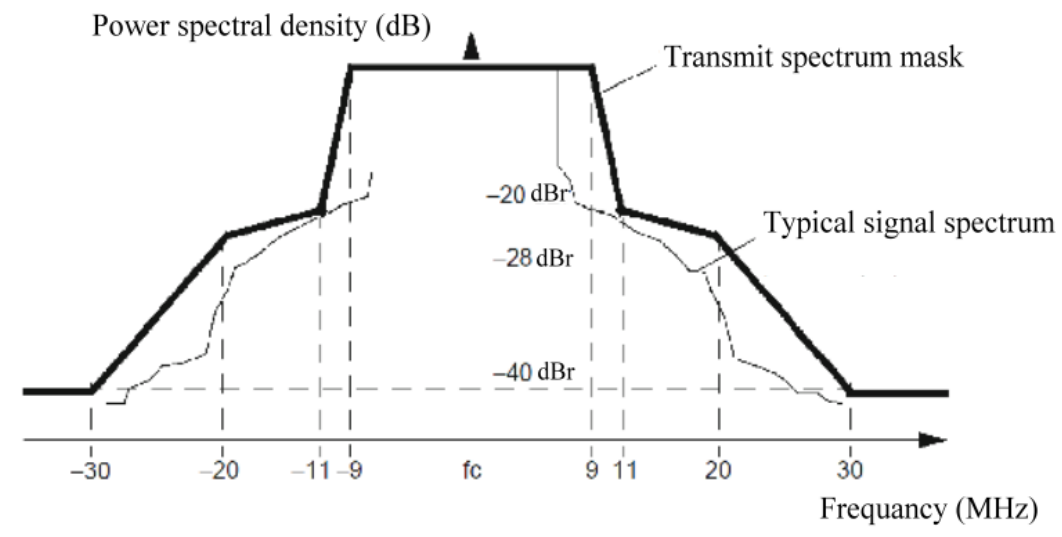

Fig. 3. Transmission spectrum mask for Wi-Fi signal

It would have been an easy task if RF filter could be used to remove any out-of-band emission at the transmitter in a wireless system. The problem is that the power amplifier is one of the major contributors to the overall chip power consumption, and the introduction of RF filter following the power amplifier would induce significant loss and decrease the chip power efficiency; therefore, it is commonly avoided in wireless systems. This places a stringent requirement to the OFDM transmitter in terms of the nonlinearity control. In contrast, in the optical systems, wavelength division multiplexing devices are commonly used to combine multiple wavelengths, and any out-ofband emission from the coherent optical-OFDM transmitter is effectively eliminated. As such, the 
coherent optical-OFDM transmitter is more tolerant to the out-of-band emission. This fact should be taken advantage of when dealing with the PAPR reduction in coherent optical-OFDM systems.

\section{Conclusions}

Communication using an optical carrier wave guided along a glass fiber has a number of extremely attractive features, several of which were apparent when the technique was originally conceived. The Optical Communications has been growing at unprecedented rates, because it is versatile and penetrates deeply into the economy, it is affecting all of society, and, therefore, has attracted inordinate amounts of public attention. The aim of this paper is illustration of advantages of optical orthogonal frequency division multiplexing in communications systems. One of the advantages of optical communication is the attenuation in fibers which could be removing without any fundamental physical effects such as Rayleigh scattering. Their prophetic prediction of $20 \mathrm{~dB} / \mathrm{km}$ for telecom-grade optical fiber was realized currently a loss of $0.2 \mathrm{~dB} / \mathrm{km}$ is the routine specification for single-mode fiber.

\section{References}

[1] Atia, W. A., Bondurant, R. S. (1999). Demonstration of return-to-zero signaling in both OOK and DPSK formats to improve receiver sensitivity in an optically preamplified receiver. Conference Paper published in 1999 IEEE LEOS Annual Meeting Conference Proceedings. LEOS'99. 12th Annual Meeting. IEEE Lasers and Electro-Optics Society 1999 Annual Meeting (Cat. No.99CH37009). doi: 10.1109/leos.1999.813561.

[2] Gnauck, A. H., Tkach, R. W., Chraplyvy, A. R., Li, T. (2008). High-capacity optical transmission systems. Journal of Lightwave Technology, Vol. 26, Issue 9, 1032-1045. doi: 10.1109/j1t.2008.922140

[3] Sano, A, Yamada, E, Masuda, H, et al. (2008). 13.4-Tb/s (134_111-Gb/s/ch) no-guard-interval coherent OFDM transmission over $3600 \mathrm{~km}$ of SMF with 19-ps average PMD. 34th European Conference on Optical Communication. doi: 10.1109/ecoc.2008.4729569

[4] Yu, J, Zhou, X, Huang, M, et al. (2008). 21.7 Tb/s (161_ 114 Gb/s) polmux-RZ-8PSK transmission over $662 \mathrm{~km}$ of ultra-low loss fiber using C-band EDFA amplification and digital coherent detection. In: Eur. Conf. Opt. Commun., paper no. Th. 3. E. 2. Brussels, Belgium.

[5] Chang, R. W. (1970). Orthogonal frequency division multiplexing. U. S. Patent no. 3488445.

[6] Toba, H, Oda, K, Inoue, K, Nosu, K, Kitoh, T. (1996). An optical FDM-based self-healing ring network employing arrayed waveguide crating filters and EDFAs with level equalizers. Journal on Selected Areas in Communications, Vol. 14, Issue 5, 800-813. doi: 10.1109/49.510904

[7] Kitayama, K. (1998). Highly spectrum efficient OFDM/PDM wireless networks by using optical SSB modulation. Journal of Lightwave Technology, Vol. 16, Issue 6, 969-976. doi: 10.1109/50.681452

[8] Shi, Q. (1997). Error performance of OFDM-QAM in subcarrier multiplexed fiber-optic transmission. IEEE Photonics Technology Letters, Vol. 9, Issue 6, 845-847. doi: 10.1109/68.585010

[9] Ma, C. P., Kuo, J. W. (2004). Orthogonal frequency division multiplex with multi-level technology in optical storage application. Japanese Journal of Applied Physics, Vol. 43, Issue 7B, 4876-4878. doi: $10.1143 /$ jjap.43.4876

[10] Dixon, B. J., Pollard, R. D., Iezekeil, S. (2001). Orthogonal frequency-division multiplexing in wireless communication systems with multimode fiber feeds. IEEE Transactions on Microwave Theory and Techniques, Vol. 49, Issue 8, 1404-1409. doi: 10.1109/22.939920

[11] Lowery, A, Armstrong, J. (2005). 10Gbit/s multimode fiber link using power-efficient orthogonal-frequency-division multiplexing. Optics Express, Vol. 13, Issue 25, 10003-10009. doi: 10.1364/opex.13.010003

[12] Shieh, W, Athaudage, C. Coherent optical orthogonal frequency division multiplexing. Electronics Letters, Vol. 42, Issue 10, 587-589. doi: 10.1049/el:20060561

[13] Mosier, R. R., Clabaugh, R. G. (1958). Kineplex, a bandwidth-efficient binary transmission system. Transactions of the American Institute of Electrical Engineers, Part I: Communication and Electronics, Vol. 76, Issue 6, 723-728. doi: 10.1109/tce.1958.6372736

[14] Tang, J. M., Lane, P. M., Shore, K. A. (2006). High-speed transmission of adaptively modulated optical OFDM signals over multimode fibers using directly modulated DFBs. Journal of Lightwave Technology, Vol. 24, Issue 1, 429-41. doi: 10.1109/j1t.2005.860146 
[15] Yi, X, Shieh, W, Ma, Y. (2007). Phase noise on coherent optical OFDM systems with 16-QAM and 64-QAM beyond $10 \mathrm{~Gb} / \mathrm{s}$. 33rd European Conference and Exhibition on Optical Communication - ECOC 2007, Berlin, Germany. doi: 10.1049/ic:20070191

[16] Takahashi, H, Amin, A. A., Jansen, S. L., Morita, I., Tanaka, H. (2008). 8×66.8-Gbit/s coherent PDM-OFDM transmission over $640 \mathrm{~km}$ of SSMF at 5.6-bit/s/Hz spectral efficiency. 34th European Conference on Optical Communication, № Th. 3. E. 4. Brussels, Belgium. doi: 10.1109/ecoc.2008.4729572

[17] Hara, S., Prasad, R. (2003). Multicarrier Techniques for 4G Mobile Communications. Boston: Artech House.

[18] Hanzo, L., Munster, M., Choi, B. J., Keller T. (2003). OFDM and MC-CDMA for Broadband Multi-User Communications, WLANs and Broadcasting. New York: Wiley. doi: 10.1002/9780470861813

[19] Shieh, W., Yi, X., Ma, Y., Tang, Y. (2007). Theoretical and experimental study on PMD-supported transmission using polarization diversity in coherent optical OFDM systems. Optics Express, Vol. 15, Issue 16, 9936-9947. doi: 10.1364/oe.15.009936

[20] Gisin, N., Huttner, B. (1997). Combined effects of polarization mode dispersion and polarization dependent losses in optical fibers. Optics Communications, Vol. 142, Issue 1-3, 119-125. doi: 10.1016/s00304018(97)00236-8

[21] Liu, X, Buchali, F. (2008). Intra-symbol frequency-domain averaging based channel estimation for coherent optical OFDM. Optics Express, Vol. 16, Issue 26, 21944-21957. doi: 10.1364/oe.16.021944

[22] Supplement to IEEE standard for information technology telecommunications and information exchange between systems-local and metropolitan area networks-specific requirements. Part 11: Wireless LAN. 